\title{
Dynamic Provisioning Utilizing Redundant Modules in Elastic Optical Networks Based on Architecture on Demand Nodes
}

\author{
Ajmal Muhammad ${ }^{(1)}$, Marija Furdek ${ }^{(2),(3)}$, Paolo Monti( ${ }^{(2)}$, Lena Wosinska ${ }^{(2)}$, Robert Forchheimer $^{(1)}$
}

(1) Department of Electrical Engineering, Linköping University, Linköping, Sweden, ajmal@isy.liu.se

(2) School of Information and Communication Technology, KTH Royal Institute of Technology, Stockholm, Sweden

(3) Faculty of Electrical Engineering and Computing, Dept. of Telecommunications, University of Zagreb, Croatia

Abstract Survivable synthetic ROADMs are equipped with redundant switching modules to support failure recovery. The paper proposes a dynamic connection provisioning strategy which exploits these idle redundant modules to provision regular traffic resulting in a substantial improvement in the blocking performance.

\section{Introduction}

In order to efficiently accommodate bandwidthintensive and dynamic network applications, as well as legacy low bitrate demands, the network infrastructure, and in particular optical nodes, need to be flexible, costeffective and reliable. Among different proposed solutions for elastic nodes, the ones based on Architecture on Demand (AoD) ${ }^{1}$ exhibits remarkable flexibility compared to the existing alternatives ${ }^{2}$. The modules of an AoD node (e.g., spectrum selective switches (SSSs), optical amplifiers, etc.) are not hard-wired, but can be connected to any other module or input/output port according to the traffic requirements. Component interconnections are provided by a high-port-count optical backplane, e.g., 3D MEMS. Thus, each AoD node and a network deploying $A \circ D$ nodes as a whole act like an optical field-programmable gate array, which can be synthesized on-the-fly ${ }^{3}$.

AoD nodes support the switching of optical signals from an input port directly to their targeted output port through the optical backplane without utilizing any (de)multiplexing modules, i.e., an operation called fiber switching. This aspect of AoD has been exploited for the cost-efficient design of nodes ${ }^{4}$ and networks ${ }^{5}$. Enhancing fiber switching improves network availability, as a decrease in the number of components traversed by a connection reduces the related risk of failure. AoD nodes can also replace failed components with idle (spare) modules on-the-fly for self-healing purposes.

On the other hand, applying cost-efficient network planning techniques ${ }^{5}$ based on replacing modules with fiber switching might lead to a degradation of the network blocking performance in dynamic network conditions, where connection requests arrive and depart stochastically. In general, connection blocking in dynamic networks based on AoD nodes can be caused by two factors: $(i)$ an insufficient number of switching modules at AoD nodes which cannot support the required connectivity; and (ii) a shortage of spectrum resources along the route between the source and destination node of a connection. Blocking inflicted by the former factor can be prevented by increasing the number of switching modules in an AoD node. Due to the flexibility of AoD nodes, another option is to uti- lize modules dedicated for protection to serve connection requests that would otherwise be blocked due to the restricted port connectivity. However, such utilization of redundant components might render them unavailable for accepting traffic from failed working components within the node, which can in turn decrease connection availability.

Inspired by these observations, this paper presents a dynamic connection provisioning strategy aimed at improving blocking probability caused by the scarcity of switching modules in AoD nodes, while balancing the trade-off with connection availability. The strategy utilizes redundant switching modules deployed in a survivable AoD nodes to accommodate the requests which would otherwise been blocked. By preempting the connections established by a redundant module in order to protect connections served by a failed working module, the proposed strategy is capable of obtaining an advantageous trade-off between blocking probability and network availability.

\section{Using preemptable modules in an AoD node archi- tecture}

The concept behind the proposed connection provisioning strategy is illustrated with Fig. 1.a, showing a possible configuration of an AoD node with nodal degree 4 supporting a set of established connections, assuming that each input fiber link has five spectrum slices. The three connections at input port 1 are fed to a SSS to allow flexible spectrum switching towards their respective output ports (i.e., one connection using slices 4-5 switched to port 3 , and two connections using slice 1, and slices $2-3$, respectively, are switched to port 1). The two connections at input port 2 are both directed to output port 4 and can be forwarded via a single cross-connect operation (i.e., fiber switching). Connections at input port 3 are fed to a SSS where the one using slice 1 is locally dropped, and the one using slice 3 is sent to output port 2 . The connections at input port 4 are cross-connected directly to output port 2.

Aside from the four working SSSs needed to support the existing traffic, the node is also equipped with one redundant SSS to be used in case a working SSS fails. Suppose now that a new connection on slice 1 appears at input port 2, with output port 3 as its desti- 

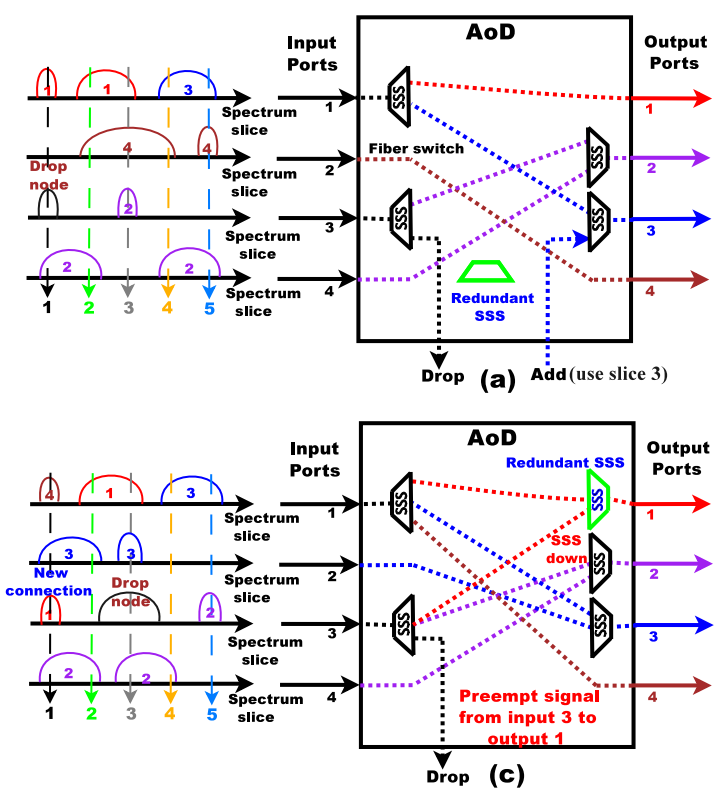
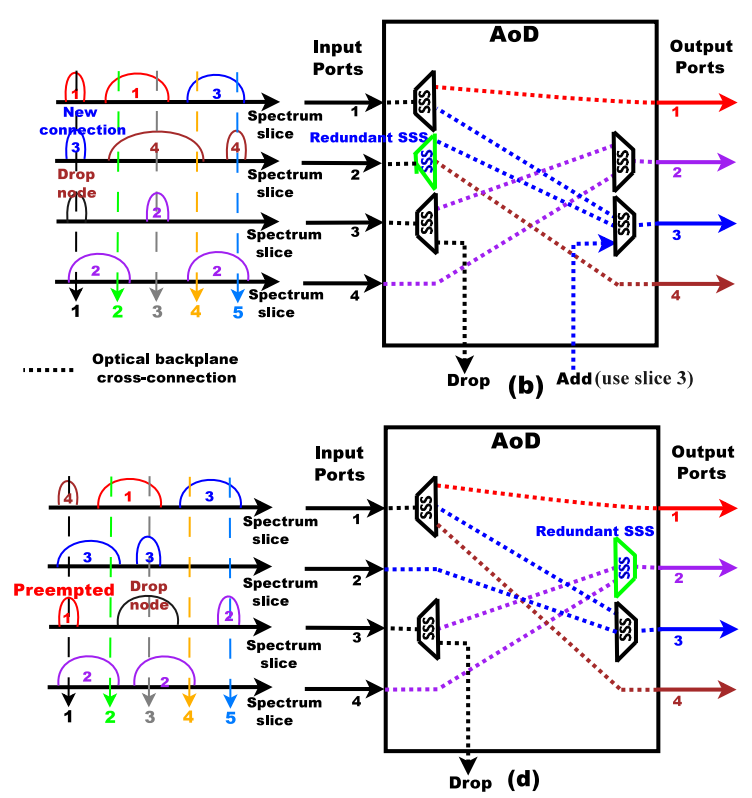

nation (Fig. 1.b). Assuming a node configuration as in Fig. 1.a, this new connection would have been blocked due to the absence of the required SSS at input port 2. However, the redundant, currently idle SSS can be put into use at input port 2 to accommodate this new connection (Fig. 1.b). If no failure happens while the spare SSS is used at input port 2, it will stay in operation as long as needed to switch the incoming traffic at input port 2 (e.g., until the connections directed to output port 4 depart from the network), after which it will go back to idle state.

Similarly, at some other time instance the redundant SSS might be used at output port 1 (Fig. 1.C) in order to multiplex a new connection from input port 3 which would otherwise have been blocked. If the working SSS at output port 2 fails while the redundant SSS is used, the redundant SSS will need to be disconnected from output port 1 and then used at output port 2. As a result connection using slice 1 at input port 3 is dropped (Fig. 1.d). Upon successful reparation of the failed SSS, the redundant module is detached from output port 2 and is put back to idle state, ready to be used again when needed. This example highlights how a redundant module in an $A 0 D$ node can be deployed to reduce the number of blocked connections at the expense of a possible degradation of connection availability. A provisioning strategy aimed at achieving a beneficial trade-off between these two objectives is presented next.

\section{Dynamic Provisioning with Preemptable Spectrum Selective Switches (DP-PSSS)}

This section describes how the concept of preemptable modules introduced in the previous section can be integrated in a dynamic provisioning strategy for AoDbased optical networks.

The connection set-up strategy presented in this paper (i.e., Dynamic Provisioning with Preemptable Spectrum Selective Switches, or DP-PSSS) works as fol- lows. Given the source and the destination node of a connection to be set up, DP-PSSS checks, for each route within a set of pre-computed $k$-shortest paths candidate, if all the AoD nodes traversed by a candidate path are capable to switch the signal from the required input to the required output port. Note that once fiber switching is used to connect an input port $i$ with an output port $j$ at a given node, it is no longer possible to switch signals from port $i$ to any output port different from $j$ as long as the connection(s) that is (are) fiber-switched between ports $i$ and $j$ are active. If the required connectivity at a given node along a candidate route is not supported due to fiber switching, DP-PSSS checks if there is an idle redundant SSS in that node. If yes, then the redundant SSS is used to switch the connection. If no spare SSSs are available, the candidate path is removed from further consideration. If there are no viable candidate paths for the current request, the connection is blocked. Otherwise, the spectrum availability is checked on each of the viable candidate paths. If free, continuous, and contiguous spectrum slices are found, the path remains a viable candidate route for provisioning the connection request. Among all the viable candidate routes, the one that requires a smaller number of new, previously unused SSSs is selected.

\section{Numerical Results}

A custom-built event-driven simulator is used to study the performance of the DP-PSSS strategy. Simulations are carried out on the NSF topology ${ }^{6}$, with 14 nodes and 42 unidirectional fiber links, each supporting 80 spectrum slices. It is assumed that only single SSS failure can occur in the network, i.e., the probability that two or more SSSs will fail at the same time is considered to be negligible. Connections are assumed to arrive in the network following a Poisson process, each one requiring a number of slices uniformly distributed among $\{2,4,6,8\}$ with an exponentially distributed duration, whose average value is set to 
1 time unit. Source/destination pairs of connection requests are assumed to be uniformly distributed among all nodes. The number of candidate paths for each connection request is set to 5 . The performance of DP-PSSS is assessed in terms of the number of demands rejected due to a limited number of switching modules at AoD nodes, and in terms of average connection availability. Results are compared against a benchmark strategy that works exactly as DP-PSSS, but does not allow using redundant SSSs to provision regular traffic. The presented results are averaged over fifty experiments for each traffic load value.

The number of active SSSs initially placed at each node is determined by the offline design procedure described $\mathrm{in}^{6}$, where the network is dimensioned differently for each specific traffic condition. This translates in an overall number of switching modules in the network that is relatively small for low loads, and increases for higher load values. Such approach avoids overprovisioning of switching modules in nodes when and where they are not needed. Finally, redundant SSSs are assumed to be placed only in those AoD nodes where at least one SSS is placed as a result of the design phase.

Fig. 2 shows the average network blocking probability for two scenarios, i.e., when AoD nodes can have at most $(i)$ one or (ii) two redundant SSSs. For each load value the dynamic provisioning of connections is simulated with the number and placement of SSSs determined in the design phase. The blocking probability of the benchmark strategy first slowly rises and then sharply declines beyond 80 Erlang. This behavior is due to the fact that at low loads the number of active SSSs placed by the design phase described above is relatively small ${ }^{5}$, yielding more restricted intra-nodal connectivity, while both parameters increase for higher load values. DP-PSSS achieves a significant reduction in blocking probability, i.e., an average gain of $53 \%$ and $62 \%$ with one and two redundant modules, respectively. The amount of spare resources depends on the load at which the network is designed. For the case of DP-PSSS with one redundant SSS, the number of spare SSSs reaches $40 \%$ of the total deployed working modules at low loads. At medium loads, this number drops to $25 \%$. On the other hand, the number of spare SSSs reaches $70 \%$ at low and $50 \%$ of total working modules at medium load conditions, respectively, for the case of DP-PSSS with two redundant SSSs.

Fig. 3 shows the average connection availability (i.e., the ratio between the time a connection is operative over the entire connection holding time) as a function of the load. The benchmark strategy, which employs the redundant resources only for failure recovery displays $100 \%$ availability. The lower blocking obtained by DPPSSS comes at the expense of slightly degraded availability due to preemption of connections which traverse a backup SSS in the event of a working module failure. However, this degradation is relatively contained,

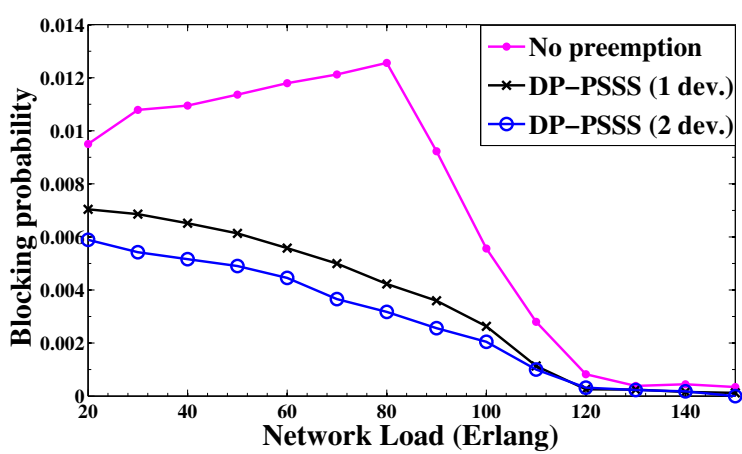

Fig. 2: Blocking probability vs. offered network load.

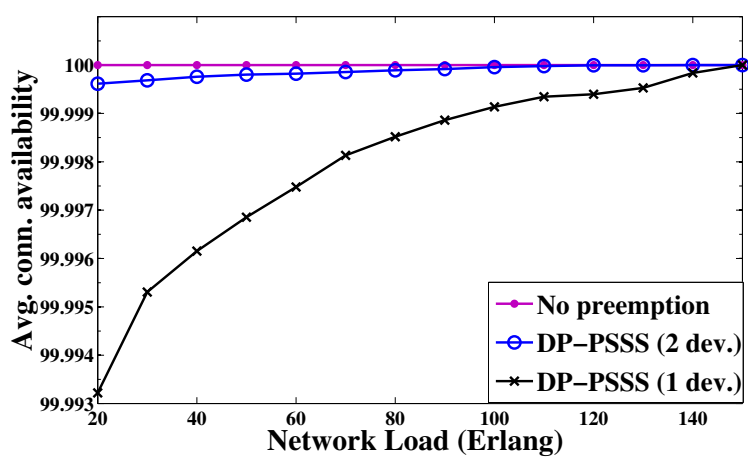

Fig. 3: Average connection availability vs. offered network load.

as it is shown that the average value of the connection availability never drops below four nines.

\section{Conclusions}

The paper presents a dynamic connection provisioning strategy for optical networks with programmable ROADMs. The proposed strategy exploits the presence of switching modules deployed for failure recovery to establish connections, which would otherwise be dropped. Simulation results confirm that the proposed approach reduces connection blocking by more than $50 \%$ without a drastic impact on the connection availability performance.

\section{Acknowledgment}

This work is supported by the EC FP7, grant agreement No. 318137 DISCUS.

\section{References}

[1] N. Amaya et al., "Architecture on demand for transparent optical networks," Proc. ICTON, Th.A1.5, (2011).

[2] Y. Wang et al., "Multi-granular optical switching: A classified overview for the past and future," IEEE com. surveys \& tutorial, (2011).

[3] N. Amaya et al., "First fully-elastic multi-granular network with space/frequency/time switching using multi-core fibres and programmable optical nodes," Proc. ECOC, Th.3.D, (2012).

[4] M. Garrich et al., "Architecture on demand: synthesis and scalability," Proc. ONDM, (2012).

[5] A. Muhammad et al., "Cost-efficient design of flexible optical networks implemented by architecture on demand," Proc. OFC, W2A.17, (2014).

[6] M. Dzanko et al., "Experimental demonstration and benefits of selfhealing hard-wired and synthetic ROADMs," Proc. OFC, W1C.3, (2014). 